\title{
Delayed presentation of visual loss from an ethmoid mucocele. Should we offer emergency decompression?
}

\section{Paula Coyle ${ }^{1}$, Andrew Dias $^{1}$}

Background

A 50 year old female presented with a 16 hour history of complete visual loss in her left eye. She had complained of blurred vision for 3 days and had seen her local ophthalmology department who were not concerned. She had no history of nasal symptoms.

\section{Method}

\section{Examination}

She had full range of eye movements with no pain and no proptosis. She had a small bruise at the medial canthus and some puffiness of upper eye lid medially. Flexible Nasendoscopy showed a large septal perforation, bulging left ethmoid bulla, but no polyps or discharge.

Imaging
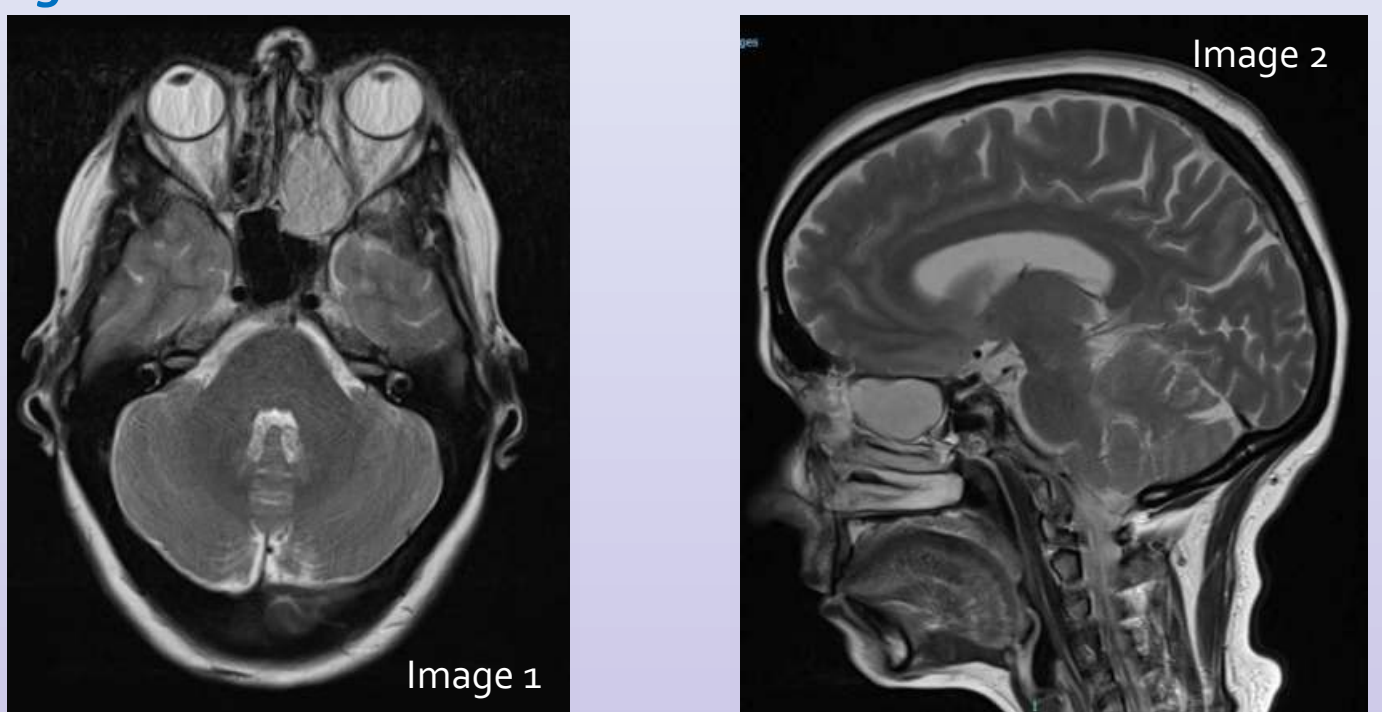

Image 1 and 2 : MRI Head - performed privately at patient's request. Showing left ethmoid mucocele.
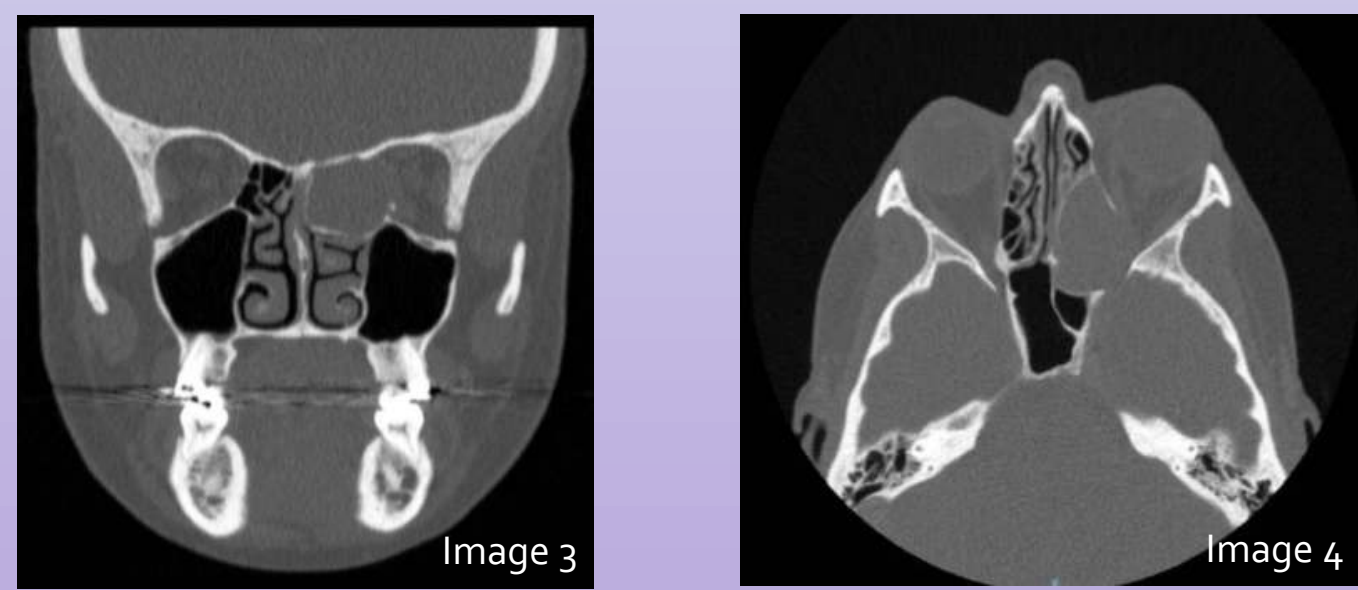

Image 3 and 4 : CT Sinus - Large left-sided ethmoid mucocele with destruction of left optic canal and compromise of the left optic nerve.

\section{Treatment}

We had a frank conversation with the patient about the likelihood of surgery being successful. The patient wished us to try and therefore she had an emergency functional endoscopic sinus surgery and decompression of the ethmoid mucocele immediately on our CEPOD list.

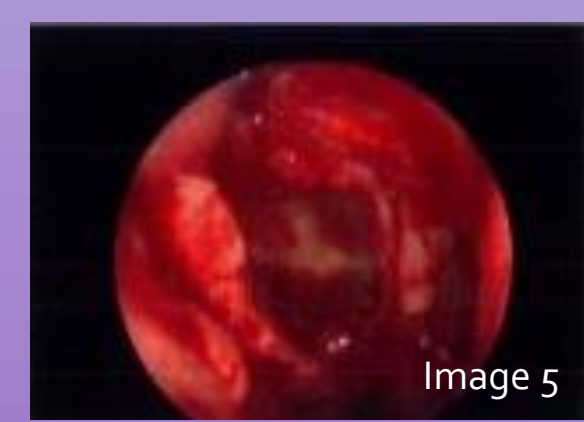

Image 5 : Intraoperative photograph after

marsupialisation of the

mucocele to the skull base.

Post operatively she was commenced on IV Cefriaxone, IV steroids, nasal steroids, douching, Otrivine and analgesia. The swab taken intraoperatively grew Staphylococcus Aureus therefore an additional 10 days of IV Flucoxicillin was commenced.

Results

Ophthalmology

Unfortunately we did not have out of hours ophthalmology cover and so our first formal assessment was post operative. At this time her Ichihara score was: Right eye 21/11 and Left eye $0 / 11$. Her Visual Acuity score was: Right eye. $6 / 5$ and Left eye 6/60. Subjectively the patient felt a 50-60\% improvement of her vision postoperatively, especially in the peripheries.

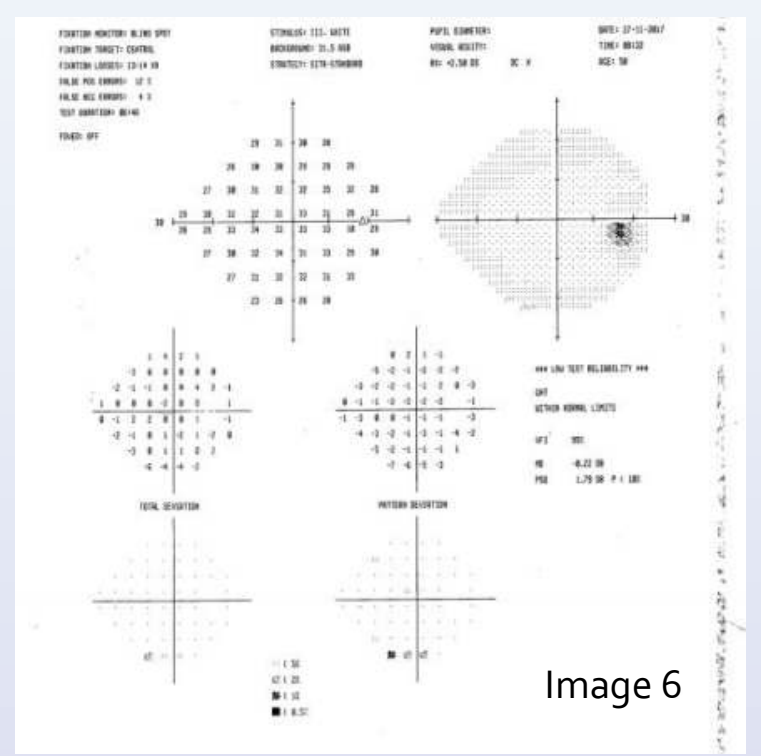

1. Ent Department, Luton and Dunstable University Hospital

Image 6 and 7 : Visual Field Test Results. 1 day post surgery. Image $6=$ Right Eye, Image $7=$ Left Eye. The darker the colour the poorer the vision.

She had monthly outpatient reviews with Ophthalmology. They placed her on a reducing dose of prednisolone, vitamin $\mathrm{D}$, calcium and omeprazole. We continued her nasal douching. Four months postoperatively she was discharged from Ophthalmology with stable vision. Her visual acuity score was: Right eye $6 / 5$ and Left eye 6/36. Her main concern was depth perception which she was reassured, she would compensate for over the next 3-4 months.

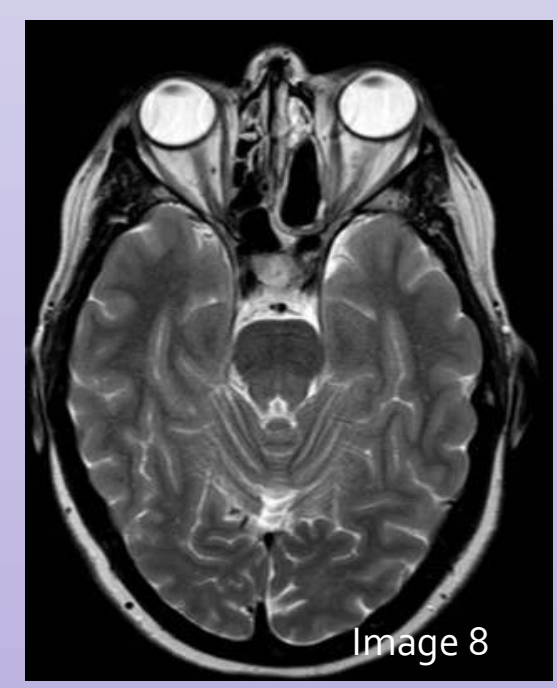

Image 8 : MRI Sinus with contrast performed one month post surgery by Ophthalmology. Showing resolution of the ethmoidal mucocele. There is increased signal in left medial rectus and adjacent fat which is likely reactive. The optic nerve and rest of extraocular

muscles are normal in appearance.

Discussion

Ethmoid Sinuses are paired sinuses varied in size and number. They lie between the nasal septum and orbit divided by the thin lamina papyracea.

Mucoceles are a collection of sinus secretions trapped due to obstruction of a sinus outflow tract. It is an expansile process. They can occur in all sinuses and are often secondary to trauma or surgery, but can have no cause. Nasal symptoms include nasal obstruction or headaches. Eye signs include proptosis, pain, reduced range of movement and eyelid swelling.

We did not have a formal ophthalmology opinion prior to performing the surgery as it was out of hours. To proceed was contentious, but after discussing thoroughly with the patient and documenting it, we collectively decided to proceed.

Surgery is the treatment of choice and most are done endoscopically ${ }^{1,2}$ but an open approach can be used. General guidelines for surgery for mucoceles are, - Identify skull base posteriorly

- Marsupialise widely, removing all osteitic bone from the opening

- Make the opening flush with the surrounding bone.

Traditional teaching suggests that optic nerve damage is permanent after 90 minutes 4,5 . Our case however shows that a proactive approach can improve symptoms significantly even at a late stage.

We conclude compressive optic neuropathy should have surgical intervention immediately even if visual loss has been sustained. 